\title{
Téoros
}

Revue de recherche en tourisme

\section{La place du marché dans le fonctionnement des loisirs sportifs de nature}

\section{Jean Corneloup}

Volume 24, numéro 1, printemps 2005

URI : https://id.erudit.org/iderudit/1071112ar

DOI : https://doi.org/10.7202/1071112ar

Aller au sommaire du numéro

Éditeur(s)

Université du Québec à Montréal

ISSN

0712-8657 (imprimé)

1923-2705 (numérique)

Découvrir la revue

Citer cet article

Corneloup, J. (2005). La place du marché dans le fonctionnement des loisirs sportifs de nature. Téoros, 24(1), 55-62. https://doi.org/10.7202/1071112ar d'utilisation que vous pouvez consulter en ligne.

https://apropos.erudit.org/fr/usagers/politique-dutilisation/ 


\section{La place du marché dans le fonctionnement des loisirs sportifs de nature}

\section{Jean Corneloup}

À une époque où la notion de marché semble s'imposer dans l'approche des activités sociales et culturelles, il apparaît judicieux d'interroger cette notion dans l'étude des loisirs sportifs de nature. Notre exposé se donne pour objectif de présenter au préalable les limites à cette entrée économique concernant ce secteur de pratique, à partir du moment où le plein air n'est pas qu'un produit, que les pratiquants ne sont pas tous des clients et que les données d'observation ne peuvent se réduire à une connaissance commerciale. Dès lors, la manière d'aborder la relation avec le pratiquant ne se définit pas de la même façon selon les acteurs engagés dans la gestion et le développement des pratiques sportives de nature et la conception qu'ils ont de l'observation et du plein air. Une autre approche des pratiques peut être saisie à partir de l'entrée par les territoires touristiques; de même, la lecture des pratiques du public permet de montrer la diversité des secteurs de pratique dans lesquels ils sont engagés. Bref, la vision économiste de la filière des loisirs sportifs de nature reste bien réductrice pour en comprendre les principes de fonctionnement.

\section{Le marché dans les pratiques sportives de nature}

Le développement des sports de nature progresse dans le monde et de nombreux indicateurs permettent d'observer ce phénomène. Sur le plan de l'offre, l'équipement de la nature est exponentiel, la création d'associations se multiplie, les distributeurs de matériels sont de plus en plus nombreux et leur chiffre d'affaires augmente constamment. Pour prendre un autre exemple, on dira que les prestataires en loisirs sportifs ne sont pas en reste de cette dynamique. En parallèle, on observe un engouement des citadins pour la vie en milieu rural, un attrait pour les pratiques sportives de nature durant les temps de loisirs et un succès remarquable des rencontres sportives en plein air (informelles et formelles), que ce soit en tant que spectateur ou pratiquant. Bref, le temps semble loin où ce secteur était accaparé par quelques passionnés, aventuriers, guides et institutionnels. Les enjeux sont aujourd'hui considérables aussi bien sur le plan économique pour de nombreuses entreprises et sur le plan institutionnel pour les différentes fédérations du plein air, que sur le plan territorial pour la plupart des régions. La marchandisation forte des sports de nature est bien dans l'air du temps. Elle a commencé avec la mise en place des différents plans neige et mer dans les années 1960 en France; elle se poursuit aujourd'hui avec les nombreux aménagements et créations de produits qui émergent ici ou là. La valeur économique des espaces de nature a connu un transfert (dans certains cas) et une bonification (dans d'autres) par la mise en marché des sports de nature en ces lieux.

Fort de ce constat, on pourrait raisonnablement penser que toutes les pratiques sportives de nature sont entrées dans l'univers du marché et qu'elles se présentent comme des produits, comme dans bien d'autres secteurs. On consomme la nature, on achète une prestation, on vend une destination, on crée de nouveaux services pour satisfaire une clientèle de plus en plus exigeante. Cependant, il faut bien apporter une correction à cette lecture à partir du moment où cette mise en marché reste bien relative. Si l'on peut penser que les grands gagnants de cette marchandisation sont les fabricants et les distributeurs de matériel (tableau 1), à partir du moment où toute pratique nécessite du matériel, on est loin d'une marchandisation totale de cette activité. Pour prendre quelques exemples, on dira que, globalement, l'espace de nature reste libre et non payant et que l'accès à de nombreux sites reste gratuit: la mer, les lacs, les canyons, les chemins de randonnée, les voies vertes, les falaises d'escalade permettent une pratique libertine et libertaire, soit en tant que passager clandestin (par refus de payer une licence fédérale), soit par libre disposition des lieux de pratique. Ajoutons que ces pratiques libres ne sont pas pour autant absentes d'enjeux autour des multiples petits défis, des prises de risque, des compétitions que se livrent les pratiquants entre eux...

En observant d'une perspective encore plus fine l'univers des pratiques, on peut facilement se rendre compte que les activités les plus pratiquées en nature s'inscrivent en dehors de toute prestation. Les différentes formes de randonnées estivales et hivernales, les activités aquatiques (baignade, planche à voile, surf...), la spéléologie, l'alpinisme, le parapente, les cyclo-pratiques, les pratiques de course à pied (trail, cross, jogging), les activités motorisées... sont réalisées en toute liberté et sans payer une quelconque prestation financière. Elles constituent la majorité des activités de loisirs sportifs de nature. Elles génèrent sans nul doute des retombées indirectes pour l'hôtellerie et la restauration, lors des déplacements dans le contexte de courts et de longs séjours, mais au regard du temps de pratique global réalisé à proximité de son domicile ou sur des sites touristiques, la part marchande reste insignifiante. Bref, le propos consiste ainsi à soutenir l'idée que le marché n'occupe qu'une faible part par rapport à l'ensemble des pratiques réalisées au quotidien ou sous forme touristique. Les pratiques en stations sportives de montagne qui proposent un produit global en hiver se présentent de ce fait comme une exception qui n'a pas son équivalent durant la saison estivale et les au- 
tres saisons. De nombreux opérateurs dans les grandes stations de ski ferment ou souhaitent interrompre leurs activités durant l'été en raison du manque de retombées financières. Bref, la dynamique du loisir s'inscrit dans son ensemble sur un espace non marchand, si l'on prend comme élément d'étude la fréquentation des lieux d'activité par le pratiquant.

Si ce constat permet de relativiser la place du marché dans l'univers des loisirs sportifs de nature, a contrario il apparaît évident que le chiffre d'affaires généré par ces consommations sportives et les activités associées est en nette croissance. Les études réalisées par B. Michon et F. Ohl (1989) sur le coût des pratiques chez les Français montrent bien que les loisirs sportifs de nature coûtent chers et que l'achat de matériel ainsi que le déplacement sur les sites occasionnent des dépenses importantes. La tendance ne va pas en s'inversant si l'on observe la prodigieuse progression du marché des équipements sportifs de nature, la technicisation exponentielle des pratiques et la tendance à la multipratique qui nécessitent à chaque fois du matériel adapté et spécifique. De même, les aménagements qui induisent des pratiques payan- tes sont de plus en plus nombreux (via ferrata $^{1}$, parcs sportifs de nature, espaces ludiques, événementiels, bassins d'eaux vives), les locations de matériels s'accroissent (kayak, VTT, matériel de canyon et de via ferrata...) et l'offre de produits proposés par les prestataires ne diminue certes pas.

À cela il faudrait ajouter la transformation des logiques territoriales qui modifient le rapport aux espaces. Si, jusque dans les années 1980, le loisir sportif de nature concernait le plein air et le grand air, le fait marquant des vingt dernières années est la forte augmen-

\section{Tableau 1}

Quelques secteurs de la filière du loisir et du tourisme sportif de nature

\begin{tabular}{|c|c|c|c|c|}
\hline Secteur & Caractéristiques & Exemples d'activités & Exemples de lieux & Acteurs impliqués \\
\hline 1 & $\begin{array}{l}\text { Pratiques libres et gratuites } \\
\text { dans un lieu de nature }\end{array}$ & $\begin{array}{l}\text { Kayak, randonnées multiples, } \\
\text { VTT, voile, aventure outdoor, } \\
\text { parapente... }\end{array}$ & Mer, lacs, forêts, chemins ruraux... & $\begin{array}{l}\text { Fabricants, distributeurs, médias } \\
\text { (hôtellerie, commerçants en option) }\end{array}$ \\
\hline 2 & $\begin{array}{l}\text { Pratiques libres et gratuites } \\
\text { sur site aménagé }\end{array}$ & $\begin{array}{l}\text { Escalade, via ferrata, canyon, } \\
\text { randonnée, plage... }\end{array}$ & $\begin{array}{l}\text { La plupart des falaises, des via ferrata, } \\
\text { des aménagements nature, } \\
\text { des bords de plage ouverts à tous... }\end{array}$ & $\begin{array}{l}\text { Fabricants, distributeurs, médias } \\
\text { (hôtellerie, commerçants en option) } \\
\text { Fédération, collectivités territoriales, } \\
\text { éco-acteurs (en option) }\end{array}$ \\
\hline 3 & $\begin{array}{l}\text { Pratiques libres et payantes } \\
\text { sur site faiblement aménagé }\end{array}$ & $\begin{array}{l}\text { Golf, parc sportif ou ludique, } \\
\text { via ferrata }\end{array}$ & Multiples sites & $\begin{array}{l}\text { Fabricants, distributeurs, médias } \\
\text { (hôtellerie, commerçants en option) } \\
\text { Fédération, collectivités territoriales, } \\
\text { éco-acteurs (en option) } \\
\text { Prestataires d'équipements }\end{array}$ \\
\hline 4 & $\begin{array}{l}\text { Pratiques libres et payantes } \\
\text { en station sportive }\end{array}$ & $\begin{array}{l}\text { Ski, surf, VTT... } \\
\text { pratiques in et arounddoor }\end{array}$ & $\begin{array}{l}\text { Les stations de ski, stations balnéaires et } \\
\text { rurales en émergence }\end{array}$ & $\begin{array}{l}\text { Fabricants, distributeurs, médias } \\
\text { (hôtellerie, commerçants en option) } \\
\text { Fédération, collectivités territoriales, } \\
\text { éco-acteurs (en option) } \\
\text { Prestataires d'équipements }\end{array}$ \\
\hline 5 & $\begin{array}{l}\text { Prestations encadrées et } \\
\text { payantes (pluri-nature) }\end{array}$ & Pratiques in, around et outdoor & $\begin{array}{l}\text { Multiples prestataires } \\
\text { (hôtellerie, commerçants en option) }\end{array}$ & $\begin{array}{l}\text { Fabricants, distributeurs, médias } \\
\text { Fédération, collectivités territoriales, } \\
\text { éco-acteurs (en option) } \\
\text { Prestataires d'équipements (en option) } \\
\text { Guides, moniteurs, autres B.E (Brevet d'État) }\end{array}$ \\
\hline 6 & $\begin{array}{l}\text { Activités encadrées en club, } \\
\text { centre local et école }\end{array}$ & Pratiques around et outdoor & $\begin{array}{l}\text { CAF (Club alpin français), FFME } \\
\text { (Fédérationfrançaise de l'escalade et de } \\
\text { la montagne),FFS (Fédération française } \\
\text { de ski)... }\end{array}$ & $\begin{array}{l}\text { Fabricants, distributeurs, médias } \\
\text { (hôtellerie, commerçants en option) } \\
\text { Clubs de loisirs locaux et autres+ fédérations } \\
\text { en option, éducateurs, enseignants, éco-acteurs } \\
\text { Guides, moniteurs, autres B.E (en option). }\end{array}$ \\
\hline 7 & $\begin{array}{l}\text { Produits intégrés en centres } \\
\text { touristiques }\end{array}$ & Pratiques in, around et outdoor & $\begin{array}{l}\text { UCPA (Union des centres de plein air), } \\
\text { Club med, Maeva, VVF (Villages vacances } \\
\text { familles), UFCV (Union française des } \\
\text { centres de vacances)... }\end{array}$ & $\begin{array}{l}\text { Fabricants, distributeurs, médias (hôtellerie, } \\
\text { commerçants en option) } \\
\text { Centre de loisir spécialisé } \\
\text { Guides, moniteurs, autres B.E (en option) }\end{array}$ \\
\hline 8 & $\begin{array}{l}\text { Pratiques compétitives } \\
\text { en club fédéral }\end{array}$ & Toutes pratiques fédérales & $\begin{array}{l}\text { Les différentes fédérations sportives } \\
\text { de nature }\end{array}$ & $\begin{array}{l}\text { Fabricants, distributeurs, médias } \\
\text { (hôtellerie, commerçants) } \\
\text { Clubs et fédérations }\end{array}$ \\
\hline
\end{tabular}


tation des pratiques de proximité sur les territoires de pratique. P. Bourdeau et P. Mao (2003) ont bien montré ce processus en montagne estivale, concernant les relations entre la haute montagne et la vallée, lorsqu'ils observent une descente progressive des pratiques vers le bas des vallées, à l'exemple du secteur de la Vallouise dans le Briançonnais. II semble ainsi possible de spatialiser la pratique en quatre cercles concentriques autour d'un site touristique de nature: l'indoor, l'arounddoor, l'outdoor et le wilddoor ${ }^{2}$, le phénomène marquant étant la forte excroissance des pratiques indoor (à l'intérieur du site) et arounddoor (à la périphérie) caractérisées par leur marchandisation. La poursuite de «l'urbanisation» des pratiques au sein des espaces de nature est d'actualité, modifiant par-là les usages de la nature sur les lieux de vacances et les pratiques professionnelles. La station des Arcs en France est un bel exemple parmi d'autres de cette situation où l'activité commerciale générée par ces pratiques (cirque, escalade, activités de forme, piscine, golf, tennis...) est largement dominante, produisant un effet géographique remarquable sur l'identité de ce site de nature.

Enfin, si l'on aborde le secteur des centres de vacances structurés par de nombreux opérateurs $^{3}$ (par ex. : UCPA, Club Med, Val, WF, UFCV, centres aérés, C.-E. ${ }^{4}$ ) et toutes les formes collectives de pratiques encadrées (séminaires d'entreprises, événements, compétitions fédérales, stations de voile, écoles de ski, clubs de sport...), on ne peut qu'observer la place centrale occupée par le marché des loisirs sportifs. C'est dans ce secteur que la marchandisation des pratiques est la plus forte sur le plan des prestations proposées ; il constitue la forme la plus intégrée du management de l'action sportive autour duquel se jouent la réussite des structures et le développement des territoires de pratique.

Pour conclure cette partie, le propos consiste à montrer que la dynamique du plein air fonctionne sur l'ambiguïté perpétuelle construite sur l'interface entre le formel et l'informel, le marchand et le non marchand, le payant et le gratuit. Si la part des pratiques instituées et marchandes progressent, il en est de même des pratiques sauvages, réalisées de manière autonome, en dehors des formes institutionnelles. La multiplication des types de pratique se généralise aujourd'hui, permettant le passage entre ces deux univers en fonction des pratiques, des moments de l'année et des formes sociales d'activité (seul/e, en groupe, en famille...). Cette logique du mixage est d'autant plus présente dans ces espaces de l'entre-deux, à l'exemple des stations de sports d'hiver et des autres stations sportives de nature en devenir, qui laissent une forte place à l'action autonome de l'individu dans sa pratique ${ }^{5}$. En échange d'une prestation de remontées mécaniques (par exemple), le pratiquant est libre de son activité et de composer lui-même sa territorialité sportive... Bref, se pose la question de la capacité à construire une lecture globale qui intègre les spécificités du loisir sportif de nature.

\section{Les secteurs du loisir et du tourisme sportifs de nature}

Pour dépasser les approches globales qui ne permettent pas une analyse affinée et diversifiée, on peut envisager de considérer la filière des loisirs sportifs de nature comme étant composée de secteurs qui participent à spécifier la relation à la nature, au marché et à l'institution. Des variables telles que l'accès libre ou payant, l'encadrement ou non et l'organisation territoriale faible ou forte permettent de qualifier les secteurs de pratique qui composent l'espace des loisirs sportifs de nature. Le ski de randonnée en individuel s'inscrit ainsi dans le secteur des pratiques libres, gratuites et faiblement aménagées, à la différence d'une prestation en via ferrata qui s'inscrit dans le secteur des pratiques encadrées, payantes et fortement aménagées. Bien souvent, la caractéristique de ces secteurs dépend de la présence ou non des acteurs du loisir sportif qui œuvrent à son organisation et à son développement (fabricant, fédération, collectivités, association, prestataires...). Pour synthétiser la lecture de cette filière, on peut présenter différents secteurs qui illustrent cette segmentation (tableau 1).

\section{La part relative du marché}

Pour approfondir le propos, il serait possible de présenter l'histoire et la dynamique intra et inter-secteurs en observant les caractéristiques de ce développement. Chaque secteur a sa propre autonomie, sa réalité et ses formes de fonctionnement. Dans bien des cas, une organisation globale par secteur n'existe pas, mais des liens inter-acteurs, des syndicats, des partenariats ou encore des fédérations participent à les structurer.
À l'exemple des clubs sportifs (fédérations de plein air), des prestations sportives (syndicats des brevetés d'État) ou des fabricants (fédérations des industries du sport), on observe une organisation d'acteurs ou de secteurs de la filière. Des liens intrasectoriels existent aussi entre, par exemple, les fédérations, les opérateurs touristiques et les fabricants. De même, il est possible de noter la présence de liens inter-sectoriels qui participent à la construction de réseaux d'acteurs, comme par exemple les liens qui existent dans les secteurs 4 et 5 entre les prestataires d'équipements (services des remontées mécaniques) et les prestataires de services sportifs (écoles de ski).

L'affinement de la notion de filière pourrait être poursuivi pour arriver à mieux identifier la valeur financière de ces secteurs, leur poids en termes de fréquentation et leur organisation. On pourrait même aller jusqu'à montrer la présence de cultures sectorielles qui participent à définir le produit, l'activité, les services ou encore la vision du client et de l'usager... En continuité avec la partie précédente, on peut faire l'hypothèse que les secteurs 1 et 2 sont sûrement les plus fréquentés par le public. Quant aux acteurs, leur poids n'est pas uniforme par secteur. II faudrait ainsi prendre en compte des différences pour relativiser l'importance donnée à certains acteurs par rapport à d'autres dans la dynamique du plein air et pour évaluer plus finement la place des différents acteurs politiques, territoriaux, fédéraux ou économiques dans la gestion de cette filière. II serait intéressant, par exemple, d'évaluer la place des acteurs issus de l'institution publique, des associations et des collectivités locales dans la gestion des secteurs et, d'une manière plus générale, dans la gestion globale de la filière.

Dans la poursuite du propos, il est possible de noter une différence entre les acteurs fortement investis dans l'aménagement et le développement et ceux qui proposent des prestations sur les aménagements publics, associatifs et fédéraux réalisés dans un secteur donné. L'entretien des chemins de randonnée, la construction de routes d'accès, l'aménagement des bords de lacs, l'équipement de sites (escalade, via ferrata, canyon...), le développement d'animations locales, la gestion des refuges sont quelques exemples d'activités d'aménagement en fonctionnement ou en investissement réalisées par des acteurs institutionnels, asso- 
ciatifs ou individuels qui profitent à des professionnels du tourisme et du loisir sportifs. Les logiques d'action des acteurs en loisir sportif ne se réduisent donc pas à une entrée par le marché pour en comprendre la valeur. Des intérêts et des modes de gestion divergents sont observables et participent à produire des écarts intra et inter-secteurs et des externalités géographiques et sectorielles (positives ou négatives). Celles-ci sont aujourd'hui l'enjeu de multiples tensions à l'image du financement des secours ou de certains équipements et accès sportifs qui produisent des controverses sociales.

Une telle lecture de cette filière par le biais des secteurs de pratique permet de relativiser la place du marché définie sous la forme d'un jeu d'échanges libres et uniformes entre une offre et une demande ${ }^{6}$. Des économies divergentes et des jeux complexes de liens et de régulation apparaissent pour nous éloigner d'une approche fordiste et instrumentale de cette filière. Les références à la sociologie économique permettent de montrer la présence d'une «économie de qualité » (Chiffoleau et Laporte, 2004) qui participe à l'organisation des échanges marchands autour d'un flou créé par l'incertitude de la valeur des biens. Dans une économie de biens immatériels (prestations touristiques par exemple) et matériels (équipements sportifs divers), la définition de la valeur du bien s'inscrit dans un jeu social au sein duquel interviennent de multiples intermédiaires ${ }^{7}$, médiations, codes professionnels et évaluations différentielles du bien par le pratiquant en fonction de son positionnement dans l'activité. La notion de champ (Bourdieu, 1987) se présente ainsi comme étant extrêmement pertinente pour montrer le rôle de l'espace des positions sociales, des réseaux sociaux, des cultures professionnelles et des effets de statut (Polanyi, 1994) dans la manière dont se construisent les secteurs sportifs. Sous un autre angle, les travaux de Bouhaouala (2002) permettent de montrer comment s'organisent les jeux professionnels dans une région de montagne, ce qui nous éloigne d'une vision économiste du marché et de la prestation. Une organisation sociale du jeu d'acteurs participe largement à bouleverser la vision parfaite de celui-ci que l'étude des micro-mentalités permet d'observer. La détermination du prix, du rapport commercial à la pratique et du produit ne se comprend pas par rapport à une logique de marché, mais bien en fonction de la forme de développement entre- preneurial et territorial au sein de laquelle le professionnel veut se situer. On observe ainsi un encastrement de l'économique dans le social (Polanyi, 1994) et dans l'univers des cultures professionnelles (Corneloup et Bourdeau, 2002) en fonction des préférences sportives, marketing, écologiques ou encore managériales affichées.

L'économie plurielle, pour reprendre les propos de différents auteurs (Passet, 2000; Loquet, 2004), est aussi une des caractéristiques de cette filière au sein de laquelle, en plus des logiques institutionnelles, fédérales et informelles multiples que l'on peut observer, s'élabore une économie sociale et solidaire qui participe à repenser la vision du marché (Laville, 2005), du tourisme et du loisir (Les Cahiers Espaces, 2004). Le développement des PNR (parc naturel régional) en France, le tourisme associatif, les pratiques libertines, le tourisme solidaire et responsable sont quelques exemples de ces variations économiques qui viennent limiter l'entrée standard par l'économie de marché pour comprendre le fonctionnement de cette filière.

Ajoutons à cela la nécessité de prendre conscience que derrière les échanges économiques et institutionnels se profilent les cultures sportives qui possèdent leurs propres logiques et leurs propres forces d'action pour structurer cet ensemble. À la différence de certaines pratiques sportives organisées par le marché et l'institution (le football, le tennis, le ski...), le loisir sportif de nature reste marqué par un «effet culturel analogique » propre aux nouvelles pratiques «fun » (Loret, 1995), par des jeux de recomposition culturelle et stylistique en fonction de la dynamique des groupes sociaux (Hoibian, 2000) et locaux (De Léséleuc, 2004) et enfin par une dynamique d'innovation et de changements culturels (Bourdeau et Mao, 2003). Le marché ne contrôle que partiellement la dynamique des loisirs sportifs de nature; il est immergé dans l'espace des cultures sportives de nature qui possède sa propre logique d'action et de fonctionnement. Les pratiques de l'escalade sont ainsi traversées par des univers culturels (les alpins-cafistes, les californiens, les hédo-sportifs, les néo-aventuriers...) qui participent à organiser les jeux de sens et les logiques sociales au sein de cette filière avec pour effet de produire un marquage culturel des biens de consommation (Corneloup, 2003). Les cultures sportives de nature s'approprient les biens de consommation pour en faire des biens cultu- rels et des objets-signes en fonction des logiques sociales engagées. Le détour par le marketing culturel (Corneloup, 2004b) s'impose ainsi aux acteurs économiques parce que le marché n'est qu'un élément d'intermédiation entre l'usager et l'espace des loisirs sportifs de nature. Une autre entrée permet de montrer que l'acte de consommation dans les loisirs sportifs de nature ne peut se réduire à un simple échange marchand, que ce soit dans le cadre d'une prestation avec un guide de montagne (Bourdeau, 1991), un vendeur chez Décathlon (Ohl et Tribou, 2004) ou dans le cadre des marchés « intermédiés » de l'occasion à l'image des trocs de matériels sportifs (Chantelat, 2004). Bref, ces auteurs positionnés en sociologie économique tentent de montrer la manière dont les actes et les objets de consommation s'inscrivent dans des interactions sociales en référence à l'interactionnisme symbolique de Goffmann (1974) pour dépasser la lecture instrumentale de l'échange marchand.

\section{La force d'action territoriale}

C'est aussi à l'échelle territoriale que s'observe cette réalité sectorielle quand territoire et marché combinent leur action dans le développement du loisir sportif de nature. Là encore, le marché n'est pas la seule entrée possible pour analyser le fonctionnement de cette filière. Le territoire, pensé comme un système touristique local (Marcelpoil et Perret, 1999), participe à structurer le ou les secteurs qui se développent sur les sites de pratique. Des effets territoriaux viennent ainsi bousculer la logique du marché par secteur de pratique. Les enjeux se compliquent évidemment lorsque plusieurs secteurs sont présents sur un même espace de pratique (pratiques libres, libres et payantes, encadrées sur parcours aménagé...). Dès lors se pose la question des liens existants entre les différents acteurs qui agissent sur un même territoire, permettant de définir l'espace commun partagé autour duquel se construit la régulation des échanges entre les différentes parties prenantes. À l'exemple des stations de sports d'hiver régies par une SEM (société d'économie mixte), une combinaison des actions publiques / privées est observable sans que soit clairement définie la construction financière et politique de cet "édifice touristique ". La question de la gouvernance (Gerbaux et Paillet, 1999) est ainsi évoquée dans la manière d'envisager la gestion des secteurs de pratique en fonction de l'ancrage territorial envisagé. 
Sans doute, peut-on penser que la présence de plusieurs formes de développement (Corneloup et al., 2001) défendues par les acteurs (qui n'ont pas le même poids) sur un territoire de pratique rend délicate la définition de cet espace commun légitime sur un territoire donné. Ce qui est en cause, ce n'est pas seulement le jeu des secteurs entre eux, mais la manière de concevoir le développement de ces secteurs. Des questions d'ordre écologique, économique, sportive et politique sont posées qui ne s'inscrivent pas dans le même univers de conception du loisir sportif de nature et dans le même champ des valeurs en fonction des secteurs, des acteurs et des publics présents localement. La diversité des territoires de pratique est bien une caractéristique de cette filière. Dans les années à venir, la combinaison territoire, marché et secteur deviendra de plus en plus pertinente à cause de la transformation des logiques territoriales, professionnelles, institutionnelles ou encore économiques qui s'inscrivent de plus en plus dans des configurations complexes de liens (Debarbieux, Vanier, 2002). Or, à suivre les propos de Pecqueur (2004), on peut penser que le détour par une géographie économique et culturelle attentive à ces effets territoriaux est de circonstance pour mieux saisir les dynamiques culturelles qui viennent bousculer la vision rationnelle du marché des loisirs sportifs de nature.

La transmodernité en émergence suggère qu'il faille savoir gérer transversalement les dynamiques territoriales et s'inscrire dans des territoires de projet au sein desquels le marché n'apparaît pas comme l'unique principe de construction des liens et des échanges. Dans ces configurations en émergence, la part du patrimoine immatériel et matériel, des ressources culturelles et des jeux socio-politiques autour desquels se définissent les identités territoriales et les logiques d'action des entrepreneurs (Bouhaouala, 2002) ne peut être sous-estimée dans la manière dont s'élabore l'orientation économique d'une destination touristique. La valeur des produits et des prestations proposés sur un site par un acteur économique dépend pour une part de cette valeur territoriale locale avec laquelle celui-ci est lié ; valeur située qui dépend de ce bien collectif partagé entre les différentes parties prenantes d'un lieu.

\section{Prolongement}

À la prise en compte de tous ces éléments (pratiques libertines, dynamique sociocultu- relle, organisation sociale du marché et effet territorial) qui viennent perturber la vision marchande de ces pratiques et de cette filière, on pourrait en ajouter deux autres :

- D'une part, il faut reconnaître la présence d'un secteur scolaire, éducatif (les mouvements de jeunesse) et social qui participe largement à la dynamique du plein air que l'on aurait tort de sous-estimer. En relation avec le développement d'une autre conception de l'économie (culture économique et sociale, tourisme diffus, culture écologique et alternative du plein air...), ce secteur, qui s'inscrit dans l'ombre de la dynamique conjoncturelle, développe une autre vision des loisirs, de la nature et de l'Autre en relation avec différentes associations de nature (FRAPNA - Fédération Rhône-Alpes de protection de la nature, Wilderness association, Mouvement d'éducation à l'environnement...). Épousant les thèses du développement durable, une remise en cause de la logique classique du marché et des territoires est perceptible et pourrait, dans les années à venir, s'imposer comme une force de développement importante. Le monde des éducateurs, des acteurs en éco-loisirs et des enseignants participe largement à la socialisation et à la construction de cette filière. Ajoutons que, selon les pays, le poids des secteurs n'est pas identique à l'image de la gestion du plein air au Québec où l'on s'aperçoit que le secteur éducatif est fortement présent et visible, à la différence de la France, avec cette volonté de transmettre une forte éducation au plein air.

- D'autre part, la relation filière-marché peut se trouver bousculée par la résurgence de la dynamique institutionnelle et étatique en France qui se perçoit, par exemple, dans des collectivités territoriales (conseil général, CROS, DDJS, CDT ... ${ }^{8}$ ) par la mise en place des $\mathrm{CDESI}^{\circ}$, la labellisation des produits, l'attribution de subventions, la définition de normes..., qui peut participer à réorganiser le jeu des acteurs dans le développement des loisirs sportifs de nature. Sans doute, on ne peut sous-estimer l'effet produit par l'adhésion en force des acteurs institutionnels, territoriaux et étatiques à la Charte du développement durable en France qui, par diffusion, risque de modifier les règles de fonctionnement du marché.

De cette configuration en gestation, des intérêts divergents émergeront, redonnant de l'importance à la régulation dans une perspec- tive communicationnelle (Habermas, 1989). Celle-ci redevient centrale aujourd'hui en invitant les différentes parties prenantes à participer à la définition de règles communes partagées qui prendront des orientations particulières en fonction des territoires et des secteurs de pratique et à partir du jeu des acteurs engagés dans la négociation et la définition de la pratique légitime. Pour limiter les prises de décision hasardeuses, la mise en place de scènes dialogiques (Callon et al., 2002) et la démocratisation des procédures décisionnelles s'imposent en favorisant la participation du public à la gestion des loisirs sportifs (Corneloup, 2002a). Mais cette participation publique ne va pas de soi ; elle est bien souvent contournée. Cependant, on peut penser que les enjeux de demain se situeront à ce niveau pour éviter de confier aux seuls experts (considérés comme légitimes) la définition des orientations politiques de cette filière et des espaces de pratique.

\section{La dynamique des styles de pratique}

Pour renforcer la teneur des propos précédents, notre approche consiste à montrer que les pratiquants, en plus de représenter une force culturelle d'action sur le marché (cf. supra), constituent une force politique que l'on ne peut sous-estimer dans la gestion de la filière des loisirs sportifs de nature ${ }^{10}$. En prenant l'exemple des pratiques sportives de montagne, on a pu élaborer l'espace des opinions sportives. Les lieux de pratique sont l'objet d'une appropriation différente en fonction des usagers et des formes de rapport aux pratiques, à l'Autre, à la nature et aux symboliques référentielles. Un jeu d'opposition est ainsi perceptible entre pratiquants qui ne partagent pas tous la même vision de la nature, ne sont pas tous demandeurs des mêmes rapports à l'ailleurs, à la sécurité, à l'engagement corporel... Au-delà de ces marquages, il semble nécessaire de concevoir la nature comme un espace de tension où les opinions sportives divergent dans la manière de définir la pratique légitime et l'orientation culturelle, économique et sportive de cet univers. Notre étude réalisée durant l'été $2002{ }^{11}$ permet de visualiser les structures de l'opinion sportive en montagne (figure 1). Entre ceux qui demandent davantage d'aménagements, de pratiques ludiques et festives et ceux qui souhaitent que la nature reste un espace sauvage, engagé et peu aménagé, des zones de tension sont per- 
Figure 1

Les structures de l'opinion sportive en montagne

\section{1}

\section{LUDISME - CHANGEMENT}

Plus de lieux de fêtes •

- Plus de pratiques culturelles et ludiques

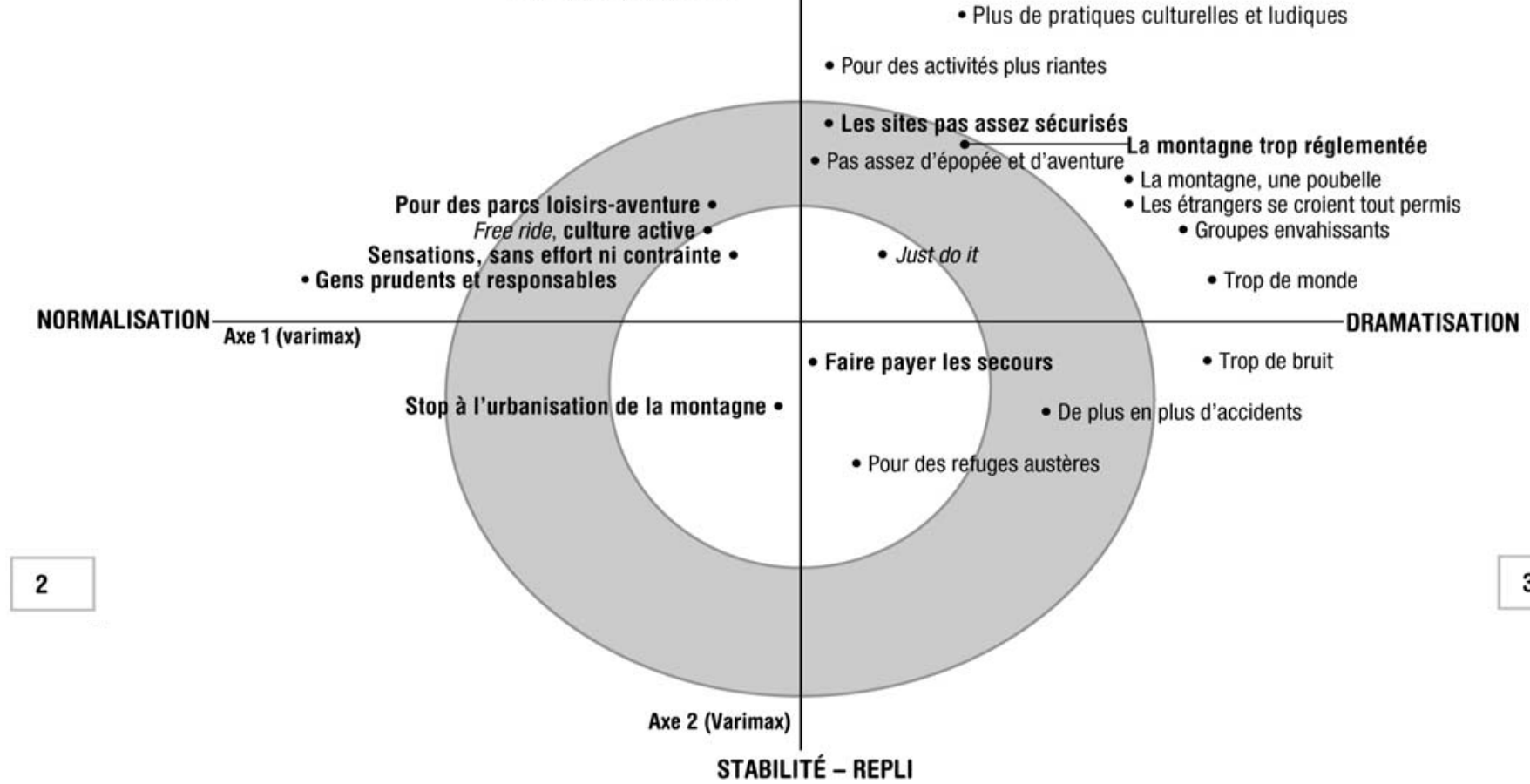

\begin{tabular}{|c|c|}
\hline $\begin{array}{l}\text { Sexe (89) • } \\
\begin{array}{c}\text { Sports classiques intensément (30) • } \\
\text { Post-matérialistes (354) } \\
\text { Débutants (158) }\end{array}\end{array}$ & 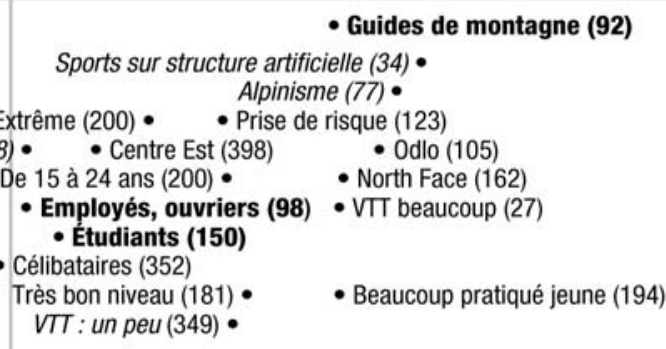 \\
\hline 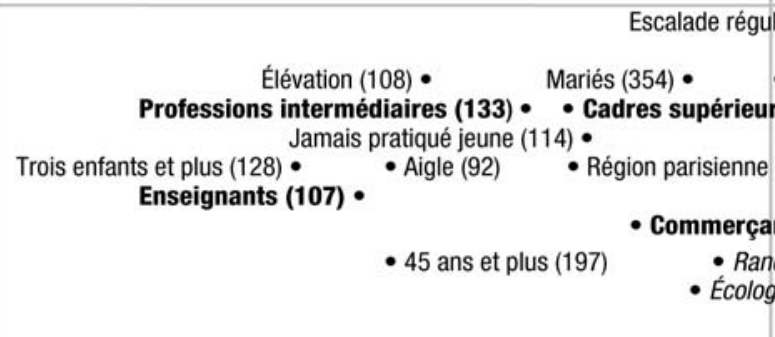 & $\begin{array}{l}\text { ulièrement (139) • • Multipratique (59) } \\
\qquad \text { Autres (155) } \\
\text { - Confirmés (207) } \\
\text { ars et professions libérales (115) } \\
\text { • Matérialiste (96) } \\
\text { (109) } \\
\text { ants, patrons de l'industrie et du commerce (22) } \\
\text { ndo (103) } \\
\text { gie et protection (74) } \\
\text { Contre la marchandisation (45) • }\end{array}$ \\
\hline
\end{tabular}

On peut en quelques mots présenter la manière de lire cette A.C.P (analyse en composantes principales). Autour des deux graphiques qui la composent: le ciel (graphique supérieur) présente le cercle de corrélation au sein duquel vont se répartir les symboles portés par les affirmations proposées dans l'enquête; la terre (graphique inférieur) correspond aux variables de la signalétique dans la perspective de préciser les liens avec le ciel par rapport à la distribution des symboles et avec les différentes variables (et indicateurs) présentées sur la terre. Schématiquement, on dira que les affirmations du quadrant 1 sur le ciel (définissant les symboles) correspondent aux positions des individus situés dans le quadrant 1 de la terre (les valeurs). Idem pour le quadrant $2 . .$. 
ceptibles. Des structures émergent qui représentent les métasymboles organisant les jeux d'opinion autour de deux axes : dramatisation / normalisation et ludisme / tradition.

L'enjeu ne consiste pas seulement à observer les jeux d'opinions entre usagers de la nature, mais à modifier la logique procédurale dans la manière de prendre des décisions. En effet, la nature est l'objet de multiples discussions concernant sa gestion. Bien souvent, on considère que seuls les experts ont les compétences requises pour prendre les bonnes décisions et définir les orientations pertinentes. Cependant, face aux multiples incohérences politiques et techniques et pour favoriser la participation du public à la gestion des lieux de pratique qu'ils affectionnent, il semble nécessaire de modifier la façon de concevoir la décision et la démocratie. Le détour par les enquêtes d'opinions permet d'observer le point de vue du pratiquant sur la gestion de la montagne, mais surtout de favoriser la présence des différentes familles politiques lors de la mise en place de procédures consultatives et dialogiques dans le contexte de discussions sur la définition de la nature d'aujourd'hui et de demain. Le développement de ce type d'enquête dans la gestion des pratiques sportives de nature serait à poursuivre en vue de renforcer la véritable « démocratisation » de ces pratiques au sens politique du terme.

En référence à notre interrogation sur la place du marché dans l'approche de cette filière, ce détour permet de montrer que le pratiquant n'est pas qu'un "simple» consommateur des produits proposés par l'offre. II représente une force d'action qui engage le marché dans une vision élargie de la filière, à partir du moment où les biens de consommation deviennent des biens politiques ${ }^{12}$. Le pratiquant, en opinant, exprime ses valeurs et sa vision de la pratique légitime, redonnant de la présence à l'espace public dans la manière de penser le développement de cet univers de pratique et des objets de consommation. Des jeux d'opposition apparaissent et des conflits émergent entre pratiquants qui interrogent sans cesse le marché, sa place et sa légitimité. À une époque où la question de la responsabilité sociétale des acteurs économiques est posée, il apparaît réducteur de considérer le marché comme étant autonome et indépendant de la sphère politique et d'un consommateur-citoyen de plus en plus actif et réactif aux désordres éthiques.

\section{Conclusion}

Aujourd'hui, le marché se présente comme un important noyau activateur dans le développement des loisirs sportifs de nature. Si cette vision instrumentale du marché irradie l'ensemble des secteurs, il ne faudrait pas pour autant réduire cette lecture à une vision simplifiée de l'économie. Des éthiques, des valeurs, des cultures professionnelles et des intérêts divergents participent à complexifier la lecture du marché et cela d'autant plus lorsque se combine l'action des grands opérateurs avec les petites entreprises et les structures en loisir sportif de nature qui ne s'inscrivent pas dans le même univers d'action. À cela, il faut ajouter la force d'action du public qui participe à la production de ce marché, se joue de lui à certains moments et n'en épouse que certaines facettes.

Par ailleurs, le propos a consisté à montrer que l'approche par le marché est insuffisante à partir du moment où le territoire est un acteur et un régulateur sectoriel qui produit une autre logique de développement. Les effets territoriaux produits s'observent lorsque émerge un marquage territorial qui impulse un style local autour d'une identité affichée, mais aussi en fonction de l'histoire des mentalités, des alliances, des tensions et des résistances locales qui agissent sur la logique du marché (Mao et al., 2003). Dès lors, face à une concurrence de plus en plus forte et à une demande sociale en transformation, la gestion territoriale des différents acteurs, parties prenantes de ce développement local, devient un enjeu pour favoriser la production d'un capital local durable (Corneloup et al., 2004). Ces deux logiques (marché et territoire) sont donc sans cesse en mouvement dans la mise en forme de la filière des loisirs sportifs de nature dans une tension perpétuelle entre le global et le local, le particulier et le général, dans une configuration rhizoïde. Dans cette conjoncture, nous avons montré que, face à la montée de ces tensions et de l'idéologie managériale (Gasparini, 2004) et pour tendre vers des décisions de meilleure qualité, la prise en compte du public et de l'opinion sportive se présente comme une démarche nécessaire dans les années à venir pour renforcer la gouvernance des territoires de pratique.

Sur un autre plan, si la conjoncture des vingt dernières années a été marquée par la force d'action du marché, on peut penser que le mouvement associatif autour de l'émergence de ce que Rifkin (2002) nomme le « tiers secteur " (économie sociale associative, coopérative et mutualiste) est un secteur en recomposition. II pourrait participer largement à la production d'une force d'action fortement active dans les années à venir, aux côtés des multiples acteurs du monde fédéral engagés sur la voie de la réforme. Cette dynamique est liée à l'émergence du mouvement écologique et alternatif, des pratiques de développement personnel et des valeurs communautaires et d'une autre approche de la responsabilité sociétale de certains acteurs de la société (Capron et Quairel, 2004 et ATTAC, 2003).

Sans doute faut-il aussi noter que la culture du plein air est de retour après le temps de la performance, de la compétition et du ludisme qui a marqué les deux décennies de la fin du vingtième siècle. On en revient à une approche globale des pratiques de nature où l'épreuve retrouve des vertus, ainsi que la contemplation, l'esthétisme, l'immersion dans la nature et toutes les pratiques d'écodéveloppement sans, pour autant, remettre en cause les principes culturels des pratiques sportives de demain (Corneloup, 2004b). Après la vague du "fun », des pratiques ludiques, des loisirs actifs de nature et des raids en tout genre, voici revenir le temps des centres et des pratiques de plein air, ces vieilles notions qui semblaient destinées à I'histoire et à l'oubli.

Jean Corneloup est maître de conférences à l'UFR STAPS de Clermont-Ferrand, membre du laboratoire de recherche Territoires (UMR PACTES - CNRS) et président du réseau des chercheurs et des experts en sports de nature (Sportsnature.org).

\section{Notes}

1 Randonnées aériennes sur grande falaise équipée de câbles ferrés pour la sécurité.

2 Nous empruntons cette notion à Philippe Bourdeau et Pascal Mao (2003) qui ont prolongé le tryptique pour spécifier le degré d'engagement dans la grande nature.

3 Au sens de Debarbieux et Marois (1997).

4 Union des centres de plein air (UCPA), Club méditerrané (Club med), Villages vacances familles (WF), Union française des centres de vacances (UFCV), Comité d'entreprise (C.-E.).

5 On a ici une spécificité française qui ne se trouve pas dans d'autres pays où une gestion plus forte des pratiques est présente avec l'existence de patrouilles des pistes par exemple.

6 L'approche par la théorie néo-classique de l'entreprise (Guerrien, 1992) et par la théorie de l'agence (Jockung-Nguéna et al., 2002) permet 
de prendre ses distances avec une vision simplifiée du marché construite sur le modèle de la microéconomie classique de la firme. Dans le cadre de la filière du loisir sportif, de nombreuses relations asymétriques, des pratiques associatives, fédérales et solidaires viennent remettre en cause la vision d'une construction de la filière par le marché.

7 Cochoy (1999) montre très bien l'impact de la médiation marchande et du marketing sur le fonctionnement du marché.

8 Comité régional olympique du sport français (CROS), comité départemental touristique (CDT), direction départementale de la jeunesse et du sport (DDJS).

9 Les Comités départementaux des espaces, sites et itinéraires (CDESI) ont été pensés dans la perspective de participer fortement à la décentralisation des différents services de l'État français.

10 Cette approche s'inscrit dans un cadre paradigmatique particulier (approche structuraliste) qui permet d'observer le social par le biais de cette focale théorique (Corneloup, 2002b).

11 L'approche théorique et méthodologique de cette enquête a été présentée dans une revue de sociologie (Corneloup, 2004).

12 Le positionnement des marques Quechua, Aigle, North Face, Odlo sur l'espace des styles de pratique permet de montrer les relations qui peuvent exister entre la consommation de biens sportifs et les valeurs exprimées. Le marché des biens de consommation s'inscrit dans un espace social plus large où se jouent d'autres jeux que les seuls jeux de la distinction et du marketing commercial.

\section{Bibliographie}

"Tourisme solidaire et responsable", (2004), Les Cahiers Espaces, Paris, Éditions Espaces, $n^{\circ} 220$.

ATTAC - Association pour la Taxation des Transactions pour l'Aide aux Citoyens (2003), "Responsabilité sociale des entreprises: faut-il y croire?", L'économie politique, $n^{0} 18,2^{\mathrm{e}}$ trimestre, Paris, Alternatives économiques.

Bouhaouala, Malek (2002), "Relations inter-entreprises dans un marché local : le cas des PE-TPE du tourisme sportif en Vercors", Espaces et Sociétés, nos 105-106, p. 229-251.

Bourdeau, Philippe (1991), "Guides de haute montagne: territoire et identité", Revue de géographie alpine, hors série, collection "Ascendances", Presses de l'Université de Grenoble, Grenoble, France.

Bourdeau, Philippe, Jean Corneloup, Pascal Mao, et Éric Boutroy (2004), «Les interactions entre cultures sportives de montagne et territoires: un état des lieux de la recherche française depuis 1990 ", Cahiers de géographie du Québec, Québec, Université Laval, vol. 48, n 133, p. 33-46.

Bourdeau, Philippe, et Pascal Mao (2002), « 'Nouvelles' pratiques sportives de nature, nouveaux espaces... nouveaux territoires? Une problématique géoculturelle de l'accès aux espaces naturels ", dans L. Mermet et P. Moquet (dir.), L'accès du public aux espaces naturels et forestiers; outils d'analyse et méthodes de gestion, Paris, HermèsLavoisier, p. 345-364.

Bourdeau, Philippe, et Pascal Mao (2003), Territoires du hors-quotidien. Une géographie culturelle du rapport à l'ailleurs dans les sociétés urbaines contemporaines, Rapport d'HDR (Habilitation à rédiger des recherches), Institut de géographie alpine, Grenoble.

Bourdieu, Pierre (1987), Choses dites, Paris, Éditions de Minuit, collection «Le sens commun".

Callon, Michel, Pierre Lascoumes, et Yannick Barthe (2002), Agir dans un monde incertain : essai sur la démocratie technique, Paris, Éditions du Seuil, collection "La couleur des idées".

Capron, M., et F. Quairel (2004), Mythes et réalités de l'entreprise responsable, Paris, La Découverte.

Chantelat, P. (2002), « La nouvelle sociologie économique et le lien marchand: des relations personnelles à l'impersonnalité des relations ", Revue française de sociologie, vol. 43, n 3, p. 521-556.

Chiffoleau, Y., et C. Laporte (2004), « La formation des prix : le marché des vins de Bourgogne », Revue française de sociologie, vol. 45, nº 4, p. 663-680.

Cochoy, F. (1999), Une histoire du marketing, Paris, La Découverte.

Corneloup, Jean (2002a), "La gestion des risques dans les sports de nature ", Les Cahier Espaces, Risques et sécurité dans le tourisme et les loisirs, Paris, Éditions Espaces, n 73, p. 32-52.

Corneloup, Jean (2003), "Les cultures sportives de la montagne d'aujourd'hui et de demain ", dans F. Bartzack et J. Rage (dir.), Les Pyrénées entre deux mondes, Perpignan, Presses de l'Université de Grenoble, Grenoble, France, p. 133-158.

Corneloup, Jean (2004a), "L'enquête d'opinion dans l'étude des pratiques sportives de montagne", Bulletin de méthodologie sociologique, $n^{\circ} 83$, p. 19-42.

Corneloup, J. (2004b), «D’hier et d'aujourd'hui. Évolution du marketing des loisirs sportifs de nature", Les Cahiers Espaces, n 81, p. 104-116.

Corneloup, J., M. Bouhaouala, C. Vachée, et B. Soulé (2001), "Formes de développement et positionnement touristique des espaces sportifs de nature ", Loisir et société, vol. 24, n 1 , p. 21-46

Corneloup, Jean, et Philippe Bourdeau (2002), "Culture professionnelle et métiers du tourisme sportif de montagne", Téoros, vol. 20, n 3 , p. 32-44.

Corneloup, Jean, Philippe Bourdeau, et Pascal Mao (2004), "Le marquage culturel des territoires sportifs de nature ", Revue de géographie alpine, $n^{\circ} 4$, p. 11-22.

Debarbieux, B., et C. Marois (1997), "Le mont Royal. Forme naturelle, paysages et territorialités urbaines ", Cahiers de géographie du Québec, Québec, Université Laval, vol. 41, no 113, p. 171-200.

Debarbieux, B., et V. Vanier (dir.) (2002), Ces territorialités qui se dessinent, La Tour d'Aigues, Éditions de l'Aube, collection «Monde en cours ».
De Léséleuc, E. (2004) «Escalade et territoire: des procédés symboliques d'appropriation d'un espace public ", Revue de géographie alpine, $\mathrm{n}^{\circ} 4$, p. 87-94.

Gasparini, W. (2004), « La face cachée de l'industrie du loisir : nouvelles formes de domination au travail dans le champ de l'offre sportive commerciale " Loisir et société, vol. 27, nº 1, p. 45-68.

Gerbaux, F., et A. Paillet (1999), "Développement local et gouvernance: enjeux et limites des recompositions territoriales ", dans F. Gerbaux Utopie pour le territoire : cohérence ou complexité ?, La Tour d'Aigues, Editions de l'Aube p. 109-142.

Goffmann, E. (1974), Les rites d'interaction, Paris, Éditions de Minuit.

Guerrien B. (1992), La théorie économique néo-classique, Paris, La Découverte.

Habermas, J. (1989), Théorie de l'agir communicationnel, Paris, Fayard.

Hoibian, O. (2000), Les alpinistes en France (1870 - 1950), une histoire culturelle, Paris, l'Harmattan.

Jockung-Nguéna, O. et al. (2002), Introduction au management de la valeur, Paris, Dunod.

Laville, J. L. (2005), Sociologie des services, Paris, Érès.

Loquet, P. (2004), L'économie sociale et solidaire au service d'un projet de territoire, Voiron, Éditions La lettre du cadre territorial, collection «Dossier d'experts".

Loret, A. (1995), Génération glisse dans l'eau, l'air, la neige: la révolution du sport des années fun, Paris, collection «Autrement ».

Mao, Pascal, Jean Corneloup, et Philippe Bourdeau (2003), "Analyse des processus de territorialisation des hauts lieux de pratiques touristiques et sportives de nature; l'exemple des Gorges du Verdon ", Téoros, vol. 22, n² 2, p. 52-62.

Marcelpoil, E., et J. Perret (1999), «Le poids conceptuel des districts industriels dans la construction des territoires", dans F. Gerbaux, Utopie pour le territoire: cohérence ou complexité? La Tour d'Aigues, Édition de l'Aube, p. 15-23.

Michon, B., et F. Ohl (1989), "Aspects socio-économiques du prix de la pratique sportive ", dans W. Andreff, Économie politique du sport, Paris, Dalloz, p. 34-72.

Ohl, F., et G. Tribou (2004), Les marchés du sport, Paris, Armand Colin.

Passet, René (2000), L'illusion néo-libérale, Paris, Fayard.

Pecqueur, B. (2004), «Vers une géographie économique et culturelle autour de la notion de territoire", Revue de géographie et cultures, nº 49, p. 71-86.

Polanyi K. (1994), The Great Transformation, Boston, Beacon Press.

Rifkin, J. (2002), L'âge de l'accès, Paris, Seuil. 